\title{
Bayesian Nash Equilibrium and Variational Inequalities
}

\author{
Takashi Ui* \\ Department of Economics \\ Hitotsubashi University \\ oui@econ.hit-u.ac.jp \\ First draft: November 2004 \\ This version: October 2015
}

\begin{abstract}
This paper provides a sufficient condition for the existence and uniqueness of a Bayesian Nash equilibrium by regarding it as a solution of a variational inequality. The payoff gradient of a game is defined as a vector whose component is a partial derivative of each player's payoff function with respect to the player's own action. If the Jacobian matrix of the payoff gradient is negative definite for each state, then a Bayesian Nash equilibrium is unique. This result unifies and generalizes the uniqueness of an equilibrium in a complete information game by Rosen (Econometrica 33: 520, 1965) and that in a team by Radner (Ann. Math. Stat. 33: 857, 1962). In a Bayesian game played on a network, the Jacobian matrix of the payoff gradient coincides with the weighted adjacency matrix of the underlying graph.
\end{abstract}

JEL classification: C72, D82.

Keywords: Bayesian game; network game; potential game; team; variational inequality; payoff gradient; strict monotonicity.

*I am very grateful for valuable input from Yasunori Kimura. I thank seminar participants at Kyoto University. I acknowledge financial support by Grant-in-Aid for Scientific Research (grant numbers 15K03348, 26245024). 


\section{Introduction}

The seminal paper by Radner (1962) is one of the earliest studies on the uniqueness of an equilibrium in Bayesian games. Radner (1962) studies a team, an identical interest Bayesian game with a common payoff function, ${ }^{1}$ and shows that if a common payoff function is strictly concave in an action profile, then a Bayesian Nash equilibrium is a unique maximizer of the payoff function. As a special case, Radner (1962) considers a linearquadratic Gaussian (LQG) team, whose payoff functions are quadratic and private signals are normally distributed, and obtains the unique equilibrium in a closed form, which is linear in private signals.

Radner's results have been widely used to study Bayesian potential games (Monderer and Shapley, 1996; van Heumen et al., 1996). A Bayesian potential game has the same best-response correspondence as that of a team, ${ }^{2}$ and the common payoff function of the team is referred to as a potential function. Because of the best-response correspondence, if a potential function is strictly concave, then a Bayesian Nash equilibrium is a unique maximizer of the potential function (Ui, 2009). Basar and Ho (1974) was the first to use Radner's results to study LQG games that are not teams, followed by many studies on information sharing in oligopoly (Clark, 1983; Vives, 1984; Gal-Or, 1985) and social value of information (Morris and Shin, 2002; Angeletos and Pavan, 2007; Ui and Yoshizawa, 2015), among others. LQG games in these studies are Bayesian potential games.

Nonetheless, we cannot rely totally on Radner's results because LQG games do not necessarily have potential functions. A payoff function in an LQG game consists of bilateral interaction terms determined by two players' actions. Such a game has a potential function if and only if every pair of players has their interaction term in common, i.e., bilateral interaction is symmetric (Ui, 2000). ${ }^{3}$ This implies that we need a new theory to study an LQG game with asymmetric interaction. For example, Calvó-Armengol et al. (2015) consider such an LQG game to analyze communication in network games and show the existence and uniqueness of a linear Bayesian Nash equilibrium. However, it has been an open question whether the linear equilibrium is a unique equilibrium.

\footnotetext{
${ }^{1}$ The theory of teams precedes Harsanyi (1967-1968).

${ }^{2} \mathrm{Ui}$ (2009) studies a game satisfying this condition and calls it a best-response Bayesian potential game.

${ }^{3} \mathrm{~A}$ game with bilateral symmetric interaction is referred to as a BSI game.
} 
This paper studies a broader class of Bayesian games, including LQG games with asymmetric interaction, and provides sufficient conditions for the existence and uniqueness of a Bayesian Nash equilibrium. To this end, we formulate a Bayesian Nash equilibrium as a solution of a variational inequality in an infinite-dimensional space (Kinderlehrer and Stampacchia, 1980), which is one representation of the first-order condition for an equilibrium. This representation not only gives us an elementary proof for the uniqueness but also allows us to use the existence theorem for solutions of variational inequalities (Browder, 1965; Hartman and Stampacchia, 1966). It is well known that a Nash equilibrium of a complete information game is a solution of a variational inequality in a finite-dimensional space (Lions and Stampacchia, 1967; Bensoussan, 1974). Thus, it is hardly surprising that a Bayesian Nash equilibrium is a solution of a variational inequality in an infinitedimensional space. To the best of the author's knowledge, however, the resulting implications are not necessarily well-documented. This paper fills this gap in the literature and shed new light on the variational inequality approach to game theory.

In the main results, we construct a vector whose component is a partial derivative of each player's payoff function with respect to the player's own action. This vector is referred to as the payoff gradient of the game. The payoff gradient is said to be strictly monotone if its Jacobian matrix is negative definite for each state. ${ }^{4}$ It is said to be strongly monotone if it is strictly monotone and the maximum eigenvalue of the Jacobian matrix has a strictly negative supremum over the actions and the states. We show that if the payoff gradient is strictly monotone, then there exists at most one equilibrium, and if the payoff gradient is strongly monotone or if it is strictly monotone and the payoff functions are quadratic, then there exists a unique equilibrium. In particular, an LQG game with asymmetric interaction has a unique equilibrium if the payoff gradient is strictly monotone.

Our condition is an extension of the sufficient condition for the uniqueness of a Nash equilibrium by Rosen (1965), who shows that a Nash equilibrium is unique if the payoff gradient of a complete information game is strictly monotone. As shown by Ui (2008), the unique Nash equilibrium is also a unique correlated equilibrium. We can show the uniqueness of a correlated equilibrium as a special case of our results because a Bayesian

${ }^{4}$ To be more precise, negative definiteness of the Jacobian matrix is a sufficient condition for strict monotonicity of the payoff gradient. 
game is reduced to a complete information game with a correlation device when payoff functions are independent of the state.

Our results generalize Radner's results and the applications to Bayesian potential games in the following sense. A Bayesian game is a Bayesian potential game if and only if the Jacobian matrix of the payoff gradient is symmetric (Monderer and Shapley, 1996), in which case the Jacobian matrix coincides with the Hessian matrix of a potential function. Thus, a potential function is strictly concave if and only if the Jacobian matrix is negative definite; that is, the payoff gradient is strictly monotone. Using this observation, we can restate Radner's results as follows: a Bayesian Nash equilibrium is unique if the Jacobian matrix is both symmetric and negative definite. We find that the symmetry condition is not necessary.

As an application, we consider games played on networks (Ballester et al., 2006; Bramoullé et al., 2014), or network games for short. ${ }^{5}$ A Bayesian game with quadratic payoff functions is mathematically equivalent to a Bayesian network game, where the Jacobian matrix of the payoff gradient equals the negative of a weighted adjacency matrix of the underlying graph. Thus, a Bayesian network game has a unique equilibrium if the weighted adjacency matrix is positive definite. This result gives a sufficient condition for the linear equilibrium in Calvó-Armengol et al. (2015) to be a unique equilibrium. Moreover, we can also use this result to study Bayesian network games with random adjacency matrices, whereas most previous studies on Bayesian network games assume a constant adjacency matrix with a special structure (Blume et al., 2015; de Martì and Zenou, 2015; Calvó-Armengol et al., 2015).

The organization of the paper is as follows. Preliminary definitions and results are summarized in Section 2. Section 3 discusses the concept of strictly monotone payoff gradients. Section 4 reports the main results. Section 5 is devoted to an application to Bayesian games played on networks.

\footnotetext{
${ }^{5}$ See Jackson and Zenou (2015) for a survey.
} 


\section{Preliminaries}

We consider a Bayesian game with a set of players $N=\{1, \ldots, n\}$. Player $i \in N$ has a set of actions $X_{i} \subseteq \mathbb{R}$, which is a closed interval. We write $X=\prod_{i \in N} X_{i}$ and $X_{-i}=\prod_{j \neq i} X_{j}$. Player $i$ 's payoff function is a measurable function $u_{i}: X \times \Omega \rightarrow \mathbb{R}$, where $(\Omega, \mathcal{F}, P)$ is a probability space. Player $i$ 's information is given by a measurable mapping $\eta_{i}: \Omega \rightarrow Y_{i}$, where $\left(Y_{i}, \mathcal{Y}_{i}\right)$ is a measurable space. Player $i$ 's strategy is a measurable mapping $\sigma_{i}$ : $Y_{i} \rightarrow X_{i}$ with $E\left[\sigma_{i}\left(\eta_{i}\right)^{2}\right]<\infty$. We regard two strategies $\sigma_{i}^{1}, \sigma_{i}^{2}$ as the same strategy if $\sigma_{i}^{1}\left(\eta_{i}(\omega)\right)=\sigma_{i}^{2}\left(\eta_{i}(\omega)\right)$ for a.e. $\omega \in \Omega$. Let $\Sigma_{i}$ denote player $i$ 's set of strategies. We write $\Sigma=\prod_{i \in N} \Sigma_{i}$ and $\Sigma_{-i}=\prod_{j \neq i} \Sigma_{j}$. We assume that $E\left[u_{i}(\sigma, \omega)\right]$ exists for all $\sigma \in \Sigma$.

We fix $N, X$, and $(\Omega, \mathcal{F}, P)$ throughout this paper and simply denote a Bayesian game by $(\mathbf{u}, \eta)$, where $\mathbf{u}=\left(u_{i}\right)_{i \in N}$ and $\eta=\left(\eta_{i}\right)_{i \in N}$. We use the following definitions and notations.

- A Bayesian game $(\mathbf{u}, \eta)$ is a team if $u_{i}=u_{j}$ for each $i, j \in N$ (Marshak, 1955; Radner, 1962; Marshak and Radner, 1972).

- A Bayesian game $(\mathbf{u}, \eta)$ is a Bayesian potential game if there exists a potential function $v: X \times \Omega \rightarrow \mathbb{R}$ such that $u_{i}\left(\left(x_{i}, x_{-i}\right), \omega\right)-u_{i}\left(\left(x_{i}^{\prime}, x_{-i}\right), \omega\right)=v\left(\left(x_{i}, x_{-i}\right), \omega\right)-$ $v\left(\left(x_{i}^{\prime}, x_{-i}\right), \omega\right)$ for each $x_{i}, x_{i}^{\prime} \in X_{i}, x_{-i} \in X_{-i}, \omega \in \Omega$, and $i \in N$ (Monderer and Shapley, 1996; van Heumen et al., 1996). The best-response correspondence of a Bayesian potential game coincides with that of a team. A Bayesian game is called a best-response Bayesian potential game if its best-response correspondence coincides with that of a team (Ui, 2009).

- A Bayesian game $(\mathbf{u}, \eta)$ is smooth if $u_{i}\left(\left(\cdot, x_{-i}\right), \omega\right): X_{i} \rightarrow \mathbb{R}$ is continuously differentiable for each $x_{-i} \in X_{-i}, \omega \in \Omega$, and $i \in N$ and $E\left[\left(\partial u_{i}(\sigma, \omega) / \partial x_{i}\right)^{2}\right]<\infty$ for each $\sigma \in \Sigma$ and $i \in N$. We write $\nabla \mathbf{u}(x, \omega) \equiv\left(\partial u_{i}(x, \omega) / \partial x_{i}\right)_{i \in N}$ and call it the payoff gradient of $\mathbf{u}$. A smooth Bayesian game $(\mathbf{u}, \eta)$ is a Bayesian potential game with a potential function $v$ if and only if the payoff gradient of $\mathbf{u}$ coincides with the gradient of $v$.

- A Bayesian game $(\mathbf{u}, \eta)$ is concave if $u_{i}\left(\left(\cdot, x_{-i}\right), \omega\right): X_{i} \rightarrow \mathbb{R}$ is concave for each $x_{-i} \in X_{-i}, \omega \in \Omega$, and $i \in N$. 
A strategy profile $\sigma \in \Sigma$ is a Bayesian Nash equilibrium if, for a.e. $\omega \in \Omega$,

$$
E\left[u_{i}(\sigma(\eta), \omega) \mid \eta_{i}\right] \geq E\left[u_{i}\left(\left(x_{i}, \sigma_{-i}\left(\eta_{-i}\right)\right), \omega\right) \mid \eta_{i}\right]
$$

for each $x_{i} \in X_{i}$ and $i \in N$, where $\sigma(\eta)=\left(\sigma_{i}\left(\eta_{i}\right)\right)_{i \in N}, \sigma_{-i}\left(\eta_{-i}\right)=\left(\sigma_{j}\left(\eta_{j}\right)\right)_{j \neq i}$, and $E\left[\cdot \mid \eta_{i}\right]$ is a conditional expectation operator given $\eta_{i}(\omega)$. We use the following representation of the first-order condition.

Lemma 1. Let $(\mathbf{u}, \eta)$ be a smooth concave Bayesian game. Then, $\sigma \in \Sigma$ is a Bayesian Nash equilibrium if and only if, for a.e. $\omega \in \Omega$,

$$
E\left[\frac{\partial}{\partial x_{i}} u_{i}(\sigma(\eta), \omega)\left(x_{i}-\sigma_{i}\left(\eta_{i}\right)\right) \mid \eta_{i}\right] \leq 0 \text { for each } x_{i} \in X_{i} \text { and } i \in N .
$$

Proof. See Appendix A.

If $\sigma \in \Sigma$ is a Bayesian Nash equilibrium, then, for a.e. $\omega \in \Omega$,

$$
E\left[\frac{\partial}{\partial x_{i}} u_{i}(\sigma(\eta), \omega)\left(\sigma_{i}^{\prime}\left(\eta_{i}\right)-\sigma_{i}\left(\eta_{i}\right)\right) \mid \eta_{i}\right] \leq 0 \text { for each } \sigma_{i}^{\prime} \in \Sigma_{i} \text { and } i \in N
$$

by Lemma 1 . By taking the expectation with respect to $\eta_{i}$, we have

$$
E\left[\frac{\partial}{\partial x_{i}} u_{i}(\sigma(\eta), \omega)\left(\sigma_{i}^{\prime}\left(\eta_{i}\right)-\sigma_{i}\left(\eta_{i}\right)\right)\right] \leq 0 \text { for each } \sigma_{i}^{\prime} \in \Sigma_{i} \text { and } i \in N
$$

By adding up the above over $i \in N$, we obtain

$$
E\left[\nabla \mathbf{u}(\sigma(\eta), \omega)^{\top}\left(\sigma^{\prime}(\eta)-\sigma(\eta)\right)\right] \leq 0 \text { for each } \sigma^{\prime} \in \Sigma,
$$

where we regard $\nabla \mathbf{u}, \sigma(\eta)$, and $\sigma^{\prime}(\eta)$ as column vectors and $x^{\top}$ denotes the transpose of a vector or a matrix $x$. The next lemma shows that this condition is not only sufficient but also necessary for a Bayesian Nash equilibrium.

Lemma 2. Let $(\mathbf{u}, \eta)$ be a smooth concave Bayesian game. Then, $\sigma \in \Sigma$ is a Bayesian Nash equilibrium if and only if (3) holds.

Proof. See Appendix A.

For example, consider a Bayesian game with quadratic payoff functions:

$$
u_{i}(x, \omega)=-q_{i i}(\omega) x_{i}^{2}-2 \sum_{j \neq i} q_{i j}(\omega) x_{i} x_{j}+2 \theta_{i}(\omega) x_{i}+h_{i}\left(x_{-i}, \omega\right)
$$


where $q_{i j}: \Omega \rightarrow \mathbb{R}$ with $q_{i i}(\omega)>0, \theta_{i}: \Omega \rightarrow \mathbb{R}$, and $h_{i}: X_{-i} \times \Omega \rightarrow \mathbb{R}$ for $i, j \in N$. We write $Q(\omega)=\left[q_{i j}(\omega)\right]_{n \times n}$ and $\theta=\left(\theta_{1}, \ldots, \theta\right)^{\top}$. Then, (3) is reduced to

$$
E\left[(Q(\omega) \sigma(\eta)-\theta)^{\top}\left(\sigma^{\prime}(\eta)-\sigma(\eta)\right)\right] \geq 0 \text { for each } \sigma^{\prime} \in \Sigma
$$

Now suppose that $Q(\omega)$ is positive definite for each $\omega \in \Omega .{ }^{6}$ Then, (5) implies the uniqueness of an equilibrium. In fact, if $\sigma^{1}, \sigma^{2} \in \Sigma$ are equilibria,

$$
\begin{aligned}
& E\left[\left(Q(\omega) \sigma^{1}(\eta)-\theta\right)^{\top}\left(\sigma^{2}(\eta)-\sigma^{1}(\eta)\right)\right] \geq 0, \\
& E\left[\left(Q(\omega) \sigma^{2}(\eta)-\theta\right)^{\top}\left(\sigma^{1}(\eta)-\sigma^{2}(\eta)\right)\right] \geq 0,
\end{aligned}
$$

which implies that

$$
E\left[\left(\sigma^{2}(\eta)-\sigma^{1}(\eta)\right)^{\top} Q(\omega)\left(\sigma^{2}(\eta)-\sigma^{1}(\eta)\right)\right] \leq 0
$$

Because $Q(\omega)$ is positive definite for each $\omega \in \Omega$, it follows that $\sigma^{1}(\eta)=\sigma^{2}(\eta)$ almost everywhere.

In the subsequent sections, we consider smooth concave Bayesian games and discuss not only the uniqueness but also the existence of Bayesian Nash equilibria on the basis of (3), which is shown to be a variational inequality.

\section{Strict monotonicity}

Let $S \subseteq \mathbb{R}^{n}$ be a convex set. A mapping $F: S \rightarrow \mathbb{R}^{n}$ is strictly monotone if $(F(x)$ $F(y))^{\top}(x-y)>0$ for each $x, y \in S$ with $x \neq y$. It is strongly monotone if there exists $c>0$ such that $(F(x)-F(y))^{\top}(x-y)>c(x-y)^{\top}(x-y)$ for each $x, y \in S$ with $x \neq y$. The following sufficient conditions are well-known. ${ }^{7}$

Lemma 3. Suppose that a mapping $F: S \rightarrow \mathbb{R}^{n}$ is continuously differentiable. If the Jacobian matrix $J_{F}(x)$ is positive definite for each $x \in S$, then $F$ is strictly monotone. There exists $c>0$ such that $x^{\top} J_{F}(x) x>c x^{\top} x$ for each $x \in S$ if and only if $F$ is strongly monotone.

\footnotetext{
${ }^{6} \mathrm{We}$ say that a square matrix $M$ is positive definite if $M+M^{\top}$ is positive definite. Note that $x^{\top} M x=$ $x^{\top}\left(M+M^{\top}\right) x / 2$.

${ }^{7}$ See Facchinei and Pang (2003), for example.
} 
With some abuse of language, we say that the payoff gradient is strictly monotone if the mapping $x \mapsto-\nabla \mathbf{u}(x, \omega)$ is strictly monotone for each $\omega \in \Omega$, i.e.,

$$
\left(\nabla \mathbf{u}(x, \omega)-\nabla \mathbf{u}\left(x^{\prime}, \omega\right)\right)^{\top}\left(x-x^{\prime}\right)<0 \text { for each } x, x^{\prime} \in X \text { with } x \neq x^{\prime} .
$$

We also say that the payoff gradient is strongly monotone if the mapping $x \mapsto-\nabla \mathbf{u}(x, \omega)$ is strongly monotone for each $\omega \in \Omega$ with respect to the same constant $c>0$, i.e.,

$$
\left(\nabla \mathbf{u}(x, \omega)-\nabla \mathbf{u}\left(x^{\prime}, \omega\right)\right)^{\top}\left(x-x^{\prime}\right)<-c\left(x-x^{\prime}\right)^{\top}\left(x-x^{\prime}\right) \text { for each } x, x^{\prime} \in X \text { with } x \neq x^{\prime} .
$$

For example, consider a Bayesian game with quadratic payoff functions (4). Because

$$
\left(\nabla \mathbf{u}(x, \omega)-\nabla \mathbf{u}\left(x^{\prime}, \omega\right)\right)^{\top}\left(x-x^{\prime}\right)=-2\left(x-x^{\prime}\right)^{\top} Q(\omega)\left(x-x^{\prime}\right),
$$

the payoff gradient is strictly monotone if and only if $Q(\omega)$ is positive definite for each $\omega \in \Omega$, and it is strongly monotone if and only if the minimum eigenvalue of $Q(\omega)$ has a strictly positive infimum over $\omega \in \Omega$.

For the general case, we have the following sufficient conditions by Lemma 3.

Lemma 4. Suppose that $\nabla \mathbf{u}(\cdot, \omega): X \rightarrow \mathbb{R}^{n}$ is continuously differentiable for each $\omega \in \Omega$. If the Jacobian matrix

$$
F_{\nabla \mathbf{u}}(x, \omega)=\left(\begin{array}{ccc}
\frac{\partial^{2} u_{1}(x, \omega)}{\partial x_{1} \partial x_{1}} & \ldots & \frac{\partial^{2} u_{1}(x, \omega)}{\partial x_{1} \partial x_{n}} \\
\vdots & \ddots & \vdots \\
\frac{\partial^{2} u_{n}(x, \omega)}{\partial x_{n} \partial x_{1}} & \ldots & \frac{\partial^{2} u_{n}(x, \omega)}{\partial x_{n} \partial x_{n}}
\end{array}\right)
$$

is negative definite for each $x \in X$ and $\omega \in \Omega$, then the payoff gradient is strictly monotone. There exists $c>0$ such that $x^{\top} F_{\nabla \mathbf{u}}(x, \omega) x<-c x^{\top} x$ for each $x \in X$ and $\omega \in \Omega$ if and only if the payoff gradient is strongly monotone.

In a complete information game (i.e. $\mathbf{u}$ is independent $\omega$ ), strict monotonicity implies not only the uniqueness of a Nash equilibrium (Rosen, 1965) but also the uniqueness of a correlated equilibrium ( $\mathrm{Ui}, 2008)$. In the next section, we show that strict monotonicity implies the uniqueness of a Bayesian Nash equilibrium and strong monotonicity implies the existence as well.

Before closing this section, we discuss two basic implications of strict monotonicity. If $F_{\nabla \mathbf{u}}(x, \omega)$ is negative definite and thus the payoff gradient is strictly monotone, $(\mathbf{u}, \eta)$ is 
concave because each diagonal element $\partial^{2} u_{i} / \partial x_{i}^{2}$ is negative. This is true even if $F_{\nabla \mathbf{u}}(x, \omega)$ does not exist (see Ui, 2008).

Lemma 5. Let $(\mathbf{u}, \eta)$ be a smooth Bayesian game. If the payoff gradient is strictly monotone, then $u_{i}\left(\left(\cdot, x_{-i}\right), \omega\right): X_{i} \rightarrow \mathbb{R}$ is strictly concave for each $x_{-i} \in X_{-i}, \omega \in \Omega$, and $i \in N$.

If $(\mathbf{u}, \eta)$ is a smooth Bayesian potential game with a potential function $v, F_{\nabla \mathbf{u}}(x, \omega)$ equals the Hessian matrix of $v$, so strictly monotonicity of the payoff gradient is equivalent to strict concavity of $v$. This is true even if $F_{\nabla \mathbf{u}}(x, \omega)$ does not exist (see Ui, 2008).

Lemma 6. Let $(\mathbf{u}, \eta)$ be a smooth Bayesian potential game with a potential function $v$. The payoff gradient is strictly monotone if and only if $v(\cdot, \omega): X \rightarrow \mathbb{R}$ is strictly concave for each $\omega \in \Omega$.

\section{Results}

First, we show that strict monotonicity is sufficient for the the uniqueness of a Bayesian Nash equilibrium.

Proposition 1. Let $(\mathbf{u}, \eta)$ be a smooth Bayesian game. Suppose that the payoff gradient is strictly monotone. Then, $(\mathbf{u}, \eta)$ has at most one Bayesian Nash equilibrium.

Proof. The proof for the uniqueness is the same as the discussion in the end of Section 2. For completeness, we give a proof. Let $\sigma^{1}, \sigma^{2} \in \Sigma$ be Bayesian Nash equilibria. Then, $\sigma^{1}$ and $\sigma^{2}$ are solutions of (3) by Lemmas 2 and 5, which implies that

$$
E\left[\nabla \mathbf{u}\left(\sigma^{1}(\eta), \omega\right)^{\top}\left(\sigma^{2}(\eta)-\sigma^{1}(\eta)\right)\right] \leq 0 \text { and } E\left[\nabla \mathbf{u}\left(\sigma^{2}(\eta), \omega\right)^{\top}\left(\sigma^{1}(\eta)-\sigma^{2}(\eta)\right)\right] \leq 0
$$

and thus

$$
E\left[\left(\nabla \mathbf{u}\left(\sigma^{2}(\eta), \omega\right)-\nabla \mathbf{u}\left(\sigma^{1}(\eta), \omega\right)\right)^{\top}\left(\sigma^{2}(\eta)-\sigma^{1}(\eta)\right)\right] \geq 0 .
$$

Strict monotonicity implies that

$$
\left(\nabla \mathbf{u}\left(\sigma^{2}(\eta), \omega\right)-\nabla \mathbf{u}\left(\sigma^{1}(\eta), \omega\right)\right)^{\top}\left(\sigma^{2}(\eta)-\sigma^{1}(\eta)\right)\left\{\begin{array}{l}
<0 \text { if } \sigma(\eta) \neq \sigma^{\prime}(\eta), \\
=0 \text { if } \sigma(\eta)=\sigma^{\prime}(\eta) .
\end{array}\right.
$$

Therefore, we must have $\sigma(\eta)=\sigma^{\prime}(\eta)$ almost everywhere. 
Using Proposition 1, we provide sufficient conditions for the existence and uniqueness of a Bayesian Nash equilibrium. In particular, strong monotonicity is a sufficient condition. Proposition 2. Let $(\mathbf{u}, \eta)$ be a smooth Bayesian game. Suppose that the payoff gradient is strictly monotone. If $X$ is bounded, or there exists $\sigma^{0} \in \Sigma$ such that

$$
\lim _{\sigma \in \Sigma, E\left[\sigma^{\top} \sigma\right] \rightarrow \infty} \frac{E\left[\nabla \mathbf{u}(\sigma(\eta), \omega)^{\top}\left(\sigma(\eta)-\sigma^{0}(\eta)\right)\right]}{\sqrt{E\left[\sigma(\eta)^{\top} \sigma(\eta)\right]}}=-\infty,
$$

then a unique Bayesian Nash equilibrium exists. If the payoff gradient is strongly monotone, then (8) is true.

To give a proof, we regard a Bayesian Nash equilibrium as a solution of a variational inequality in an infinite-dimensional space (see Stampacchia, 1970; Kinderlehrer and Stampacchia, 1980). As shown by Lions and Stampacchia (1967) and Bensoussan (1974), a Nash equilibrium of a complete information game is a solution of a variational inequality in a finite-dimensional space (see Harker and Pang, 1990; Facchinei and Pang, 2003). In the following proof, we use the fact that a Bayesian Nash equilibrium is a solution of a variational inequality in a Hilbert space.

Let $H$ be a Hilbert space with an inner product $\langle\cdot, \cdot\rangle: H \times H \rightarrow \mathbb{R}$. Fix a non-empty subset $C \subseteq H$ and let $T: C \rightarrow H$ be a mapping. A variational inequality is a problem to seek $\alpha \in C$ satisfying

$$
\left\langle T \alpha, \alpha^{\prime}-\alpha\right\rangle \geq 0 \text { for each } \alpha^{\prime} \in C
$$

The following result is due to Browder (1965) and Hartman and Stampacchia (1966). ${ }^{8}$

Proposition 3 (Browder-Hartman-Stampacchia). Assume the following conditions.

1. $C$ is a nonempty closed convex subset of $H$.

2. $\langle T \alpha-T \beta, \alpha-\beta\rangle \geq 0$ for all $\alpha, \beta \in C$.

3. The mapping $t \mapsto\langle T((1-t) \alpha+t \beta), \gamma\rangle$ from $[0,1]$ to $\mathbb{R}$ is continuous for all $\alpha, \beta \in C$ and $\gamma \in H$.

4. $C$ is bounded, or there exists $\alpha^{0} \in C$ such that

$$
\lim _{\|\alpha\| \rightarrow \infty} \frac{\left\langle T \alpha, \alpha-\alpha^{0}\right\rangle}{\|\alpha\|}=+\infty .
$$

${ }^{8}$ They consider a reflexive Banach space. 
Then, there exists $\alpha \in C$ satisfying (9).

We are ready to prove Proposition 2.

Proof of Proposition 2. Proposition 1 implies the uniqueness. To prove the existence, we show that the first-order condition (3) is a special case of the Browder-Hartman-Stampacchia variational inequalities. Let $H$ be a Hilbert space consisting of (an equivalence class of) a random variable $\alpha: \Omega \rightarrow \mathbb{R}^{n}$ with $\langle\alpha, \beta\rangle \equiv E\left[\alpha(\omega)^{\top} \beta(\omega)\right]$ for $\alpha, \beta \in H$. Let $C=\Sigma \subseteq H$, which is a nonempty closed convex subset of $H$. Let $T: C \rightarrow H$ be such that $T \sigma=-\nabla \mathbf{u}(\sigma(\eta), \omega) \in H$ for each $\sigma \in \Sigma$, which satisfies the conditions in Proposition 3. Because (9) coincides with (3), there exists $\sigma \in \Sigma$ satisfying (3) by Proposition 3, which is a Bayesian Nash equilibrium by Lemmas 2 and 5.

If the payoff gradient is strongly monotone, there exists $c>0$ such that

$$
\begin{aligned}
\nabla \mathbf{u}(\sigma, \omega)^{\top}\left(\sigma-\sigma^{0}\right) & \leq \nabla \mathbf{u}\left(\sigma^{0}, \omega\right)^{\top}\left(\sigma-\sigma^{0}\right)-c\left(\sigma-\sigma^{0}\right)^{\top}\left(\sigma-\sigma^{0}\right) \\
& =-c \sigma^{\top} \sigma+\nabla \mathbf{u}\left(\sigma^{0}, \omega\right)^{\top} \sigma+\left(c\left(2 \sigma-\sigma^{0}\right)-\nabla \mathbf{u}\left(\sigma^{0}, \omega\right)\right)^{\top} \sigma^{0}
\end{aligned}
$$

for each $\sigma, \sigma^{0} \in \Sigma$ and $\omega \in \Omega$. Thus,

$$
\begin{aligned}
& \frac{E\left[\nabla \mathbf{u}(\sigma, \omega)^{\top}\left(\sigma-\sigma^{0}\right)\right]}{\sqrt{E\left[\sigma^{\top} \sigma\right]}} \\
& =-c \sqrt{E\left[\sigma^{\top} \sigma\right]}+\frac{E\left[\nabla \mathbf{u}\left(\sigma^{0}, \omega\right)^{\top} \sigma+\left(c\left(2 \sigma-\sigma^{0}\right)-\nabla \mathbf{u}\left(\sigma^{0}, \omega\right)\right)^{\top} \sigma^{0}\right]}{\sqrt{E\left[\sigma^{\top} \sigma\right]}} .
\end{aligned}
$$

Because the second term in the above is bounded, we have (8).

In the case of quadratic payoff functions, we need neither (8) nor strong monotonicity. Strict monotonicity suffices, as the next proposition shows. In the proof, we consider another Hilbert space.

Proposition 4. Let (u, $\eta$ ) be a Bayesian game with quadratic payoff functions (4). Suppose that $Q(\omega)$ is positive definite for each $\omega \in \Omega$. Then, a unique Bayesian Nash equilibrium exists.

Proof. Proposition 1 implies the uniqueness. To prove the existence, we show that (5) is another special case of the Browder-Hartman-Stampacchia variational inequalities. Let $H$ be a Hilbert space consisting of (an equivalence class of) a random variable $\alpha: \Omega \rightarrow \mathbb{R}^{n}$ 
with $\langle\alpha, \beta\rangle \equiv E\left[\alpha(\omega)^{\top} Q(\omega)^{\top} \beta(\omega)\right]$ for $\alpha, \beta \in H$. Let $C=\Sigma \subseteq H$, which is a nonempty closed convex subset of $H$. Let $T: C \rightarrow H$ be such that $T \sigma=\sigma-Q^{-1} \theta \in H$ for each $\sigma \in \Sigma$. Note that $Q$ is invertible because $Q$ is positive definite. ${ }^{9}$ Then, (9) is written as

$$
\left\langle T \sigma, \sigma^{\prime}-\sigma\right\rangle=\left\langle\sigma-Q^{-1} \theta, \sigma^{\prime}-\sigma\right\rangle=E\left[(Q(\omega) \sigma(\eta)-\theta)^{\top}\left(\sigma^{\prime}(\eta)-\sigma(\eta)\right)\right] \geq 0
$$

for each $\sigma^{\prime} \in \Sigma$, which is (5). Thus, a Bayesian Nash equilibrium is a solution of a variational inequality (10). Because

$$
\begin{aligned}
\lim _{\|\sigma\| \rightarrow \infty} \frac{\left\langle T \sigma, \sigma-\sigma^{0}\right\rangle}{\|\sigma\|} & =\lim _{\|\sigma\| \rightarrow \infty} \frac{\left\langle\sigma-Q^{-1} \theta, \sigma-\sigma^{0}\right\rangle}{\|\sigma\|} \\
& \geq \lim _{\|\sigma\| \rightarrow \infty}\left(\|\sigma\|-\left\|\sigma^{0}\right\|-\left\|Q^{-1} \theta\right\|+\frac{\left\langle Q^{-1} \theta, \sigma^{0}\right\rangle}{\|\sigma\|}\right)=\infty,
\end{aligned}
$$

a Bayesian Nash equilibrium exists by Proposition 3.

Applying Propositions 4 to linear quadratic Gaussian (LQG) games, where $Q$ is constant and an information structure is Gaussian, we obtain the following existence and uniqueness result.

Proposition 5. Let $(\mathbf{u}, \eta)$ be a Bayesian game with quadratic payoff functions (4). Assume the following conditions.

1. $Q$ is positive definite and independent of $\omega$.

2. $X_{i}=\mathbb{R}$ and $Y_{i}=\mathbb{R}^{m_{i}}$ for each $i \in N$, where $m_{i} \geq 1$ is an integer.

3. $\eta_{1}(\omega), \ldots, \eta_{n}(\omega)$ and $\theta_{1}(\omega), \ldots, \theta_{n}(\omega)$ are jointly normally distributed with

$$
\operatorname{cov}\left[\eta_{i}, \eta_{j}\right]=C_{i j}, \operatorname{cov}\left[\eta_{i}, \theta_{i}\right]=G_{i}
$$

where $C_{i i}$ is positive definite for each $i \in N$.

Then, there exists a unique Bayesian Nash equilibrium obtained as follows:

$$
\sigma_{i}\left(\eta_{i}\right)=b_{i}^{\top}\left(\eta_{i}-E\left[\eta_{i}\right]\right)+c_{i}
$$

where $b_{i}$ and $c_{i}$ are determined by the system of linear equations

$$
\begin{gathered}
\sum_{j \in N} q_{i j} C_{i j} b_{j}=G_{i} \text { for } i \in N, \\
\sum_{j \in N} q_{i j} c_{j}=E\left[\theta_{i}\right] \text { for } i \in N .
\end{gathered}
$$

${ }^{9}$ Otherwise, there exists $x \neq \mathbf{0}$ such that $Q x=\mathbf{0}$, which implies $x^{\top} Q x=0$, a contradiction. 
Proof. Proposition 4 implies the existence and uniqueness. Thus, it is enough to show that the unique Bayesian Nash equilibrium is of the above form. See Appendix B.

In all the above results, we assume that the payoff gradient is strict monotone. However, even without strict monotonicity, we can obtain similar results if the best-response correspondence coincides with that of another game whose payoff gradient is strictly monotone. For example, for two games $(\mathbf{u}, \eta)$ and $\left(\mathbf{u}^{\prime}, \eta\right)$, if there exist $w_{i}>0$ and $h_{i}: X_{-i} \times \Omega \rightarrow \mathbb{R}$ such that $u_{i}^{\prime}(x, \omega)=w_{i} u_{i}(x, \omega)+h_{i}\left(x_{-i}, \omega\right)$ for each $x \in X, \omega \in \Omega$, and $i \in N$, then the best-response correspondences coincide. Thus, if the payoff gradient of $\mathbf{u}$ is strictly monotone, $\left(\mathbf{u}^{\prime}, \eta\right)$ has at most one Bayesian Nash equilibrium, and if the payoff gradient of $\mathbf{u}$ is strongly monotone, $\left(\mathbf{u}^{\prime}, \eta\right)$ has a unique Bayesian Nash equilibrium. ${ }^{10}$

The above discussion leads us to the following weaker concept of strict or strong monotonicity. For $w \equiv\left(w_{i}\right)_{i \in N} \in \mathbb{R}_{++}^{n}$, we call $w \circ \nabla \mathbf{u} \equiv\left(w_{i} \partial u_{i} / \partial x_{i}\right)_{i \in N}$ the $w$-weighted payoff gradient of $\mathbf{u}$. We say that the $w$-weighted payoff gradient is strictly monotone if the mapping $x \mapsto-w \circ \nabla \mathbf{u}(x, \omega)$ is strictly monotone for each $\omega \in \Omega$. We also say that the $w$-weighted payoff gradient is strongly monotone if the mapping $x \mapsto-w \circ \nabla \mathbf{u}(x, \omega)$ is strongly monotone for each $\omega \in \Omega$ with respect to the same constant $c>0$. Then, strict or strong monotonicity of the payoff gradient in Propositions 1 and 2 can be replaced with strict or strong monotonicity of the $w$-weighted payoff gradient without any change in the conclusions. The corresponding concept for complete information games is discussed by Rosen (1965). For much weaker concepts, which also work, see Ui (2008).

Remark 1. Radner (1962) and Krainak et al. (1982) consider teams and obtain a special case of Proposition 1. Radner (1962) considers teams with quadratic payoff functions as well as LQG teams and obtains special cases of Propositions 4 and 5. Ui (2009) considers best-response Bayesian potential games and obtains special cases of Propositions 1, 4, and 5 using the results of Radner (1962) and Krainak et al. (1982).

Remark 2. If $\mathbf{u}$ is independent of $\omega \in \Omega,(\mathbf{u}, \eta)$ is a complete information game with a correlation device $\eta$, and a Bayesian Nash equilibrium is a correlated equilibrium. Thus, Proposition 1 implies that if the payoff gradient is strictly monotone, a unique Nash equilibrium is a unique correlated equilibrium, which is the result obtained by Ui (2008).

${ }^{10}$ For more general conditions for best-response equivalence, see Morris and Ui (2004) and Ui (2009). 


\section{An application to network games}

Consider a Bayesian game with quadratic payoff functions (4). For each $\omega \in \Omega$, the matrix $Q(\omega)$ defines a directed graph with a set of nodes $N$ and a set of directed edges $E(\omega)=$ $\left\{(i, j): q_{i j}(\omega) \neq 0, i, j \in N\right\}$. Thus, we can regard this game as a Bayesian game played on a random network, or a Bayesian network game for short, where $Q(\omega)$ is a weighted adjacency matrix of the underlying graph. Proposition 4 states that a Bayesian network game has a unique equilibrium if the weighted adjacency matrix is positive definite for each $\omega \in \Omega$.

\section{The network game of Ballester et al. (2006)}

Ballester et al. (2006) consider a network game with complete information such that $A_{i}=$ $[0, \infty), \theta_{1}=\cdots=\theta_{n}=\alpha$, and $Q=\beta I+\gamma U-\lambda G$, where $\alpha, \beta, \gamma, \lambda>0$ are constant, $I$ is the identity matrix, $U$ is the matrix of ones, and $G=\left[g_{i j}\right]$ is a symmetric matrix with $g_{i j} \in[0,1]$ and $g_{i i}=0$. Ballester et al. (2006) show that this game has a unique Nash equilibrium if the maximum eigenvalue of $G$ is less than $\beta / \lambda$, which implies that $\beta I+\gamma U-\lambda G$ is positive definite (but not vice versa).

Now consider a Bayesian network game, where $\alpha, \beta, \gamma, \lambda>0$ are random variables and $G$ is a random asymmetric matrix with $g_{i j} \in[0,1]$ and $g_{i i}=0$. By Proposition 4 , this game has a unique Bayesian Nash equilibrium if $\beta I+\gamma U-\lambda G$ is positive definite for each state. de Martì and Zenou (2015) consider a special case of this Bayesian network game, where $\beta, \lambda>0, \gamma=0$, and $g_{i j} \in\{0,1\}$ are constant, and show that it has a unique Bayesian Nash equilibrium if the maximum eigenvalue of $G$ is less than $\beta / \lambda$.

\section{The network game of Bramoullé et al. (2014)}

Bramoullé et al. (2014) consider a network game with complete information such that $A_{i}=[0, \infty), \theta_{i}=\bar{x}_{i}, Q=I+\delta G$, where $\bar{x}_{i}, \delta>0$ are constant, $I$ is the identity matrix, and $G=\left[g_{i j}\right]$ is a symmetric matrix with $g_{i j} \in\{0,1\}$ and $g_{i i}=0$. Bramoullé et al. (2014) show that this game has a unique Nash equilibrium if the absolute value of the minimum eigenvalue of $G$ is less than $1 / \delta$, which occurs if and only if $I+\delta G$ is positive definite.

Now consider a Bayesian network game, where $\bar{x}_{i}, \delta>0$ are random variables and $G$ 
is a random asymmetric matrix with $g_{i j} \in[0,1]$ and $g_{i i}=0$. By Proposition 4 , this game has a unique Bayesian Nash equilibrium if the absolute value of the minimum eigenvalue of $G$ is less than $1 / \delta$ for each state.

\section{The network game of Blume et al. (2015)}

Blume et al. (2015) consider a Bayesian network game such that $A_{i}=\mathbb{R}, \theta_{i}, \ldots, \theta_{n}$ are random variables, and $Q=(1+\phi) I-\phi W$ is a constant matrix, where $\phi>0, I$ is the identity matrix, and $W=\left[w_{i j}\right]$ is a nonnegative matrix such that $w_{i j} \in[0,1], \sum_{j \in N} w_{i j} \in\{0,1\}$, and $w_{i i}=0$. Blume et al. (2015) show that this game has a unique Bayesian Nash equilibrium.

Now consider another Bayesian network game, where $\phi>0$ is a random variable and $W$ is a random matrix. We do not require the above condition on $W$, but assume that $(1+\phi) I-\phi W$ is positive definite for each state. Then, this game has a unique Bayesian Nash equilibrium by Proposition 4.

\section{The network game of Calvó-Armengol et al. (2015)}

Calvó-Armengol et al. (2015) use an LQG framework to study communication in network games. After exchanging information, players play an LQG game with a payoff function

$$
u_{i}(x, \omega)=-d_{i i}\left(x_{i}-\theta_{i}\right)^{2}-\sum_{j \neq i} d_{i j}\left(x_{i}-x_{j}\right)^{2}
$$

where $\theta_{i}$ is normally distributed and $d_{i j} \geq 0$ for each $i, j \in N$. Calvó-Armengol et al. (2015) obtain a unique linear equilibrium, but it has been an open question whether the linear equilibrium is a unique equilibrium.

By dividing (14) by $D_{i}=\sum_{j \in N} d_{i j}$, we obtain (4) with $Q=I-W$, where $W=\left[w_{i j}\right]$ is a nonnegative matrix with

$$
w_{i j}= \begin{cases}d_{i j} / D_{i} & \text { if } j \neq i, \\ 0 & \text { if } j=i .\end{cases}
$$

Thus, if $I-W$ is positive definite, then the linear equilibrium is a unique equilibrium by Proposition 5.11

\footnotetext{
${ }^{11}$ The operator norm of $W$ corresponding to the $\infty$-norm for vectors (i.e. $\max _{i \in N} \sum_{j \in N}\left|w_{i j}\right|$ ) is less than one, so $I-W$ is invertible as shown by Calvó-Armengol et al. (2015). The matrix $I-W$ is positive definite if and only if the operator norm of $W$ corresponding to the 2-norm for vectors is less than one.
} 


\section{Appendix}

\section{A Proof of Lemmas 1 and 2}

Proof of Lemma 1. Because $u_{i}\left(\left(\cdot, x_{-i}\right), \omega\right): X_{i} \rightarrow \mathbb{R}$ is concave,

$$
\frac{\partial}{\partial x_{i}} E\left[u_{i}\left(\left(x_{i}, \sigma_{-i}\right), \omega\right) \mid \eta_{i}\right]=E\left[\frac{\partial}{\partial x_{i}} u_{i}\left(\left(x_{i}, \sigma_{-i}\right), \omega\right) \mid \eta_{i}\right]
$$

by the Lebesgue monotone convergence theorem (see p.863 of Radner (1962)).

For $x_{i}^{\prime} \in X_{i}$, let $f(t)=E\left[u_{i}\left(\left(\sigma_{i}+t\left(x_{i}^{\prime}-\sigma_{i}\right), \sigma_{-i}\right), \omega\right) \mid \eta_{i}\right]$. If (1) is true, $f(t)$ achieves its maximum at $t=0$. Thus, it must be true that

$$
\begin{aligned}
f^{\prime}(0) & =\left.\frac{\partial}{\partial x_{i}} E\left[u_{i}\left(\left(x_{i}, \sigma_{-i}\right), \omega\right) \mid \eta_{i}\right]\right|_{x_{i}=\sigma_{i}}\left(x_{i}^{\prime}-\sigma_{i}\left(y_{i}\right)\right) \\
& =E\left[\frac{\partial}{\partial x_{i}} u_{i}(\sigma, \omega) \mid \eta_{i}\right]\left(x_{i}^{\prime}-\sigma_{i}\right) \\
& =E\left[\frac{\partial}{\partial x_{i}} u_{i}(\sigma, \omega)\left(x_{i}^{\prime}-\sigma_{i}\right) \mid \eta_{i}\right] \leq 0 .
\end{aligned}
$$

Thus, (2) is true.

Conversely, suppose that (2) is true. Note that $f(t)$ is concave in $t$ because $u_{i}\left(\left(\cdot, x_{-i}\right), \omega\right)$ : $X_{i} \rightarrow \mathbb{R}$ is concave. Thus, $f(t) \leq f(0)+t f^{\prime}(0)$ for each $t$ and

$$
\begin{aligned}
E\left[u_{i}\left(\left(x_{i}^{\prime}, \sigma_{-i}\right), \omega\right) \mid \eta_{i}\right] & =f(1) \\
& \leq f(0)+f^{\prime}(0) \\
& =E\left[u_{i}(\sigma, \omega) \mid \eta_{i}\right]+E\left[\frac{\partial}{\partial x_{i}} u_{i}(\sigma, \omega)\left(x_{i}^{\prime}-\sigma_{i}\right) \mid \eta_{i}\right] \\
& \leq E\left[u_{i}(\sigma, \omega) \mid \eta_{i}\right]
\end{aligned}
$$

by (2). Thus, (1) is true.

Proof of Lemma 2. We have already shown that if $\sigma$ is a Bayesian Nash equilibrium then (3) is true. We show that if $\sigma$ is not a Bayesian Nash equilibrium then (3) is not true. Suppose that $\sigma \in \Sigma$ is not a Bayesian Nash equilibrium. Then, by Lemma 1, there exist $j \in N, \sigma_{j}^{\prime} \in \Sigma_{j}$, and $E \subseteq \Omega$ with $P(E)>0$ such that $E\left[\partial u_{j}(\sigma, \omega) / \partial x_{j} \cdot\left(\sigma_{j}^{\prime}-\sigma_{j}\right) \mid \eta_{j}\right]>0$ for each $\omega \in E$. Let $\sigma^{\prime \prime} \in \Sigma$ be such that

$$
\sigma_{i}^{\prime \prime}\left(\eta_{i}(\omega)\right)= \begin{cases}\sigma_{i}^{\prime}\left(\eta_{i}(\omega)\right) & \text { if } i=j \text { and } \omega \in E \\ \sigma_{i}\left(\eta_{i}(\omega)\right) & \text { otherwise }\end{cases}
$$


Then,

$$
E\left[\nabla \mathbf{u}(\sigma, \omega)^{\top}\left(\sigma^{\prime}-\sigma\right)\right]=\sum_{i \in N} E\left[\partial u_{i}(\sigma, \omega) / \partial x_{i} \cdot\left(\sigma_{i}^{\prime \prime}-\sigma_{i}\right)\right]>0,
$$

so (3) is not true.

\section{B Proof of Proposition 5}

We use the following lemma (see p.870 of Radner (1962)).

Lemma A. Suppose that $C$ is a $K \times K$ symmetric positive semi-definite matrix, partitioned symmetrically into blocks $C_{i j}$, such that $C_{i i}$ is positive definite for every $i$, and that $Q$ is an $n \times n$ symmetric positive definite matrix with elements $q_{i j}$. Then, the matrix $H$ composed of blocks $q_{i j} C_{i j}$ is positive definite.

Proof of Proposition 5. Proposition 4 guarantees the existence and uniqueness of a Bayesian Nash equilibrium. We show that it is given by (11), (12), and (13).

Because $A_{i}=\mathbb{R}$ for each $i \in N$, the first-order condition for an equilibrium is

$$
\sum_{j \in N} q_{i j} E\left[\sigma_{j} \mid \eta_{i}=y_{i}\right]=E\left[\theta_{i} \mid \eta_{i}=y_{i}\right]
$$

for each $y_{i} \in Y_{i}$ and $i \in N$. If an equilibrium is of the form (11), (B1) is calculated as

$$
\sum_{j} q_{i j}\left(b_{j}^{\top} C_{j i} C_{i i}^{-1}\left(y_{i}-E\left[\eta_{i}\right]\right)+c_{j}\right)=E\left[\theta_{i}\right]+G_{i}^{\top} C_{i i}^{-1}\left(y_{i}-E\left[\eta_{i}\right]\right)
$$

for each $y_{i} \in Y_{i}$ and $i \in N$. Thus, $b_{i}$ and $c_{i}$ are determined by

$$
\sum_{j} q_{i j} b_{j}^{\top} C_{j i} C_{i i}^{-1}=G_{i}^{\top} C_{i i}^{-1} \text { and } \sum_{j} q_{i j} c_{j}=E\left[\theta_{i}\right] \text { for } i \in N,
$$

which is reduced to (12) and (13).

To complete the proof, it is enough to show that the system of linear equations (12) and (13) has a unique solution. Since $Q$ is positive definite, $Q$ is invertible, by which the solvability of (13) follows. Let $C$ be the covariance matrix of $\eta$, which satisfies the condition imposed on $C$ in Lemma A. Let $R$ be the matrix composed of blocks $q_{i j} C_{i j}$ and let $H$ be the matrix composed of blocks $\left(q_{i j}+q_{j i}\right) C_{i j}$. Since $C$ is symmetric, $H=R+R^{\top}$. By Lemma A, $H$ is positive definite. Therefore, $R$ is also positive definite and thus invertible, by which the solvability of (12) follows. 


\section{References}

Angeletos, G.-M., Pavan, A., 2007. Efficient use of information and social value of information. Econometrica 75, 1103-1142.

Ballester, C., Calvó-Armengol, A., Zenou, Y., 2006. Who's who in networks. Wanted: the key player. Econometrica 74,1403-1417.

Basar, T., Ho, Y., 1974. Informational properties of the Nash solution of the two stochastic nonzero-sum games. J. Econ. Theory 7, 370-384.

Bensoussan, A., 1974. Points de Nash dans le cas de fonctionnelles quadratiques et jeux differentiels lineaires a $N$ personnes. SIAM J. Control 12, 460-499.

Blume, L., Brock, W., Durlauf, S., Jayaraman, R., 2015. Linear social interactions models. J. Polit. Econ. 123, 444-496

Bramoullé, Y., Kranton, R., D’amours, M., 2014. Strategic interaction and networks. Amer. Econ. Rev. 104, 898-930.

Browder, F. E., 1965. Nonlinear monotone operators and convex sets in Banach spaces. Bull. Amer. Math. Soc. 71, 780-785.

Calvó-Armengol, A., de Martì, J., Prat, A., 2015. Communication and influence. Theoretical Econ. 10, 649-690.

Clark, R. 1983. Collusion and the incentives for information sharing. Bell J. Econ. 14, 383-394.

de Martì, J., Zenou, Y., 2015. Network games with incomplete information. Forthcoming in J. Math. Econ.

Facchinei, F., Pang, J. S., 2003. Finite-Dimensional Variational Inequalities and Complementarity Problems. Springer.

Gal-Or, E., 1985. Information sharing in oligopoly. Econometrica 53, 329-343.

Harker, P. T., Pang, J. S., 1990. Finite-dimensional variational inequality and nonlinear complementarity problems. Math. Programming 48, 161-220. 
Harsanyi, J. C., 1967-1968. Games with incomplete information played by Bayesian players. Manage. Sci. 14, 159-182, 320-334, 486-502.

Hartman, P., Stampacchia, G., 1966. On some nonlinear elliptic differential functional equations. Acta Math. 115, 271-310.

Jackson, M., Zenou, Y., 2015. Games on networks. In: Young, P., Zamir, S. (Eds.), Handbook of Game Theory Vol. 4. North Holland, pp. 91-157.

Kinderlehrer, D., Stampacchia, G., 1980. An Introduction to Variational Inequalities and Their Applications. SIAM.

Krainak, J. C., Speyer, J. L., Marcus, S. I., 1982. Static team problems - Part I: sufficient conditions and the exponential cost criterion. IEEE Trans. Automat. Contr. AC-27, 839848.

Lions, J. L., Stampacchia, G., 1967. Variational inequalities. Commun. Pur. Appl. Math. 20, 493-519.

Marshak, J., 1955. Elements for a theory of teams. Manage. Sci. 1, 127-137.

Marshak, J., Radner, R., 1972. Economic Theory of Teams. Yale University Press.

Monderer, D., Shapley, L. S., 1996. Potential games. Games Econ. Behav. 14, 124-143.

Morris, S., Shin, H. S., 2002. Social value of public information. Amer. Econ. Rev. 52, $1521-1534$.

Morris, S., Ui, T., 2004. Best response equivalence. Games Econ. Behav. 49, 260-287.

Radner, R., 1962. Team decision problems. Ann. Math. Statist. 33, 857-881.

Rosen, J. B., 1965. Existence and uniqueness of equilibrium points for concave $N$-person games. Econometrica 33, 520-534.

Stampacchia, G., 1970. Variational inequalities. Actes Congrés intern. Math. 2, 877-883.

Ui, T., 2000. A Shapley value representation of potential games. Games Econ. Behav. 31, $121-135$. 
Ui, T., 2008. Correlated equilibrium and concave games. Int. J. Game Theory 37, 1-13.

Ui, T., 2009. Bayesian potentials and information structures: team decision problems revisited. Int. J. Econ. Theory 5, 271-291.

Ui, T., Yoshizawa, Y., 2015. Characterizing social value of information. J. Econ. Theory 158, 507-535.

van Heumen, R., Peleg, B., Tijs, S., Borm, P., 1996. Axiomatic characterizations of solutions for Bayesian games. Theory Dec. 40, 103-130.

Vives, X., 1984. Duopoly information equilibrium: Cournot and Bertrand. J. Econ. Theory 34, 71-94. 